\title{
Extended Finite Element for Electromechanical Coupling
}

\author{
Véronique Rochus and Daniel Rixen \\ Delft University of Technology, faculty $3 \mathrm{mE}$ \\ Dpt. of Precision and Microsystems Engineering, Engineering Dynamics \\ Mekelweg 2, 2628 CD Delft, The Netherlands \\ V.Rochus@tudelft.nl, D.J.Rixen@tudelft.nl \\ Phone: +31-(0) 15-278 1881
}

\begin{abstract}
In MEMS modelling the electro-mechanical coupling takes an important place. Indeed many devices use electrostatic forces as actuator. The numerical modelling of this type of problem needs a strong coupling between the mechanics and the electrostatic field. In fact when the structure is moving, the electrostatic field around it has to be modified in consequence. The first solution is to use finite element method to model the electrostatic field. In this case the mesh has to be updated depending on the displacement of the structure. Many researches have been performed to deform properly the electrostatic mesh, but when large displacement are taken into account, the elements become distorted. Furthermore, when the pull-in is achieved, the electrodes are in contact and the layer of electrostatic elements is totally squeezed. The second usual solution is to use the boundary element method to model the electrostatic field. In this case, there are no more remeshing problem, but the computational time is larger and singularity problems appears when the electrodes become in contact.

One solution for this remeshing problem is to use extended finite elements (X-FEM) which are a new type of elements tailored to simulate problems involving discontinuities and moving boundaries. Initially this methodology was created for crack propagation problems $[1,3,5]$, but its application has been extended to several other problems such as elastic problem involving inclusions, flowstructure interaction and solidification problems. In this paper the concept of extended finite elements is applied to develop modelling approaches for the electro-mechanical coupling. The method will be illustrated here for a onedimensional problem and implementation issues relative to the two-dimensional case are discussed.
\end{abstract}

\section{Introduction}

This research aims at modelling of electro-mechanical coupling that normally takes place in some micro-electromechanical systems (MEMS) like micro-resonator or RF switches. The problem can be described as a conducting mechanical structure with applied voltage, which generates a surrounding electrostatic field. The electrostatic field, in its turn, causes an appearance of electrostatic force, applied to the structure. This type of problem is a strongly nonlinear problem since the electric domain changes with the deformation of the structure. The usual numerical techniques to model this type of electro-mechanical problem are the finite element method and the boundary element method. The mechanical structure is usually simulated by a finite element model and the electrostatic domain is solved by either finite element method or boundary element method. For both cases some problems appear when the structure undergoes large displacements and when the electrodes come into contact. Indeed, the electrostatic finite element mesh has to be modified as the structure moves. Moreover, the electrostatic mesh can be severely deformed if the structure undergoes large displacements. Furthermore, when the electrodes come into contact, the elements between the electrodes have to be deleted. The boundary element method proposes a partial solution to this problem: the electrostatic domain is meshed only on the boundary hence allowing large displacements to the structure. However this increases the computation time. Moreover, when the structure comes into contact, the boundary element can no longer be applied since it requires that a gap exists between the electrodes. In order to simplify and improve modelling of structures moving in an electric field, we propose to make use of the concept of eXtented Finite Elements. They are a new type of elements tailored to simulate problems involving discontinuities and moving boundaries.

The basic idea is to have an electrostatic mesh covering the entire domain and that does not change while the structure part is moved within the field. The electro-mechanical problem is considered as a bi-material problem where the mechanics and the electricity are computed and coupled on a single element. Following the variational approach developed in [4], electrostatic forces may be derived and applied at the interface of the element. The electromechanical problem may then be solved and the results correspond very well to the analytical solution for a one dimensional problem. A short discussion will be also given about implementation issues of this techniques in 2 dimensions.

\section{2. eXtended Finite Element Theory}

The extended finite element method consists in discretising the entire electro-mechanical problem with a fixed mesh and in following the interface between two domains through this mesh. At the interface the physical field or its gradient are no more continuous. To model this discontinuity, special shape functions are used to enrich the usual discretisation. For instance, the mechanical displacement $u$ is enhanced by discontinuous shape functions $M_{i}$ such as:

$$
\boldsymbol{u}(\boldsymbol{x}, t)=\sum_{i} N_{i}(\boldsymbol{x}) U_{i}(t)+\sum_{j} M_{j}(\boldsymbol{x}, t) A_{j}(t)
$$


where $N_{i}$ are the standard shape functions and $M_{j}$ are the enriched shape functions taking the discontinuity into account. New unknowns $A_{j}$ are introduced to model the discontinuity.

There are different ways to create these additional shape functions. Moes [2] proposes to define these functions based on the standard shape functions by the relation:

$$
M_{j}(\boldsymbol{x}, t)=N_{j}(\boldsymbol{x}) \Theta(\boldsymbol{x}, t)
$$

where $\Theta(x, t)$ is defined by:

$$
\Theta(\boldsymbol{x}, t)=\sum_{i}\left|\psi_{i}\right| N_{i}-\left|\sum_{i} \psi_{i} N_{i}\right|
$$

where $\psi(x, t)$ is the level set field describing the location of the interface, and $\psi_{i}$ is the value of the level set of the node $i$. The level set is described by a surface function intersecting the problem plan at the interface.

First this methodology will be applied in a onedimensional electro-mechanical example and the electromechanical problem will be solved by computing electrostatic forces at the interface. Then this methodology will be adapted to the two dimension problem.

\section{Implementation in One Dimension}

\subsection{Shape Functions in 1D}

In this section the shape functions for a pure mechanical bi-material element are presented in one dimension. In that case the usual shape functions $N_{i}$ on a reference element are the following:

$$
\left\{\begin{array}{l}
N_{1}=1-\eta \\
N_{2}=\eta
\end{array}\right.
$$

These shape functions are represented in Figure 1. They allows to model linear behaviour in the element.

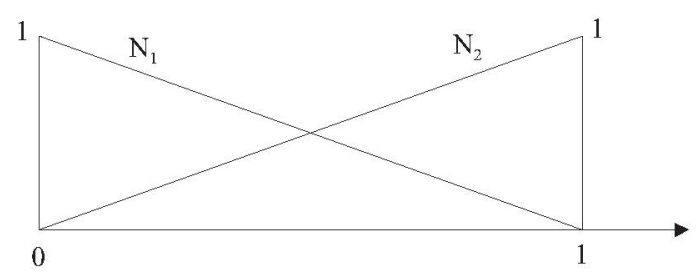

Figure 1: Linear shape functions in 1D.

Then the enriched shape functions are added to model the discontinuity. The enriched shape functions, following the approach of Moes [2], are given by (2) where $\Theta$ is defined by the level set $\Psi$ equal to the signed distance between the point $x$ and the discontinuity which is located at a distance $\Gamma$ from the first node. In the 1D model, this enrichment $\Theta$ becomes:

$$
\begin{cases}\Theta_{a}=\frac{\eta}{\Gamma} & \text { for } 0 \leq \eta \leq \Gamma \\ \Theta_{b}=\frac{1-\eta}{1-\Gamma} & \text { for } \Gamma \leq \eta \leq 1\end{cases}
$$

The plot of these functions is illustrated in Figure 2 and

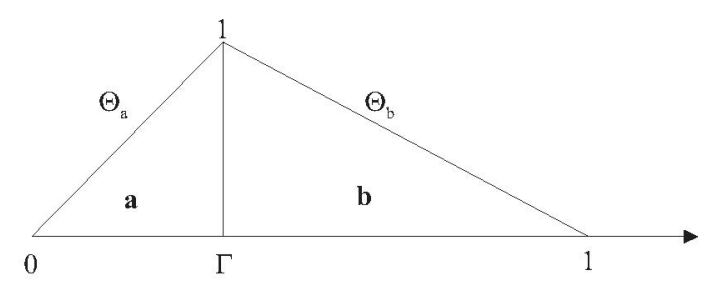

Figure 2: Extended finite element.

the enriched shape functions are:

$$
\left\{\begin{array}{l}
M_{1 a}=(1-\eta) \frac{\eta}{\Gamma} \\
M_{2 a}=\eta \frac{\eta}{\Gamma} \\
M_{1 b}=(1-\eta) \frac{1-\eta}{1-\Gamma} \\
M_{2 b}=\eta \frac{1-\eta}{1-\Gamma}
\end{array}\right.
$$

The sum of these two shape functions corresponds to the expression of $\Theta$ (see equation (5)).

$$
\left\{\begin{array}{l}
M_{1 a}+M_{2 a}=\frac{\eta}{\Gamma} \\
M_{1 b}+M_{2 b}=\frac{1-\eta}{1-\Gamma}
\end{array}\right.
$$

which correspond of the plot in Figure 2

\subsection{Application to Electro-mechanical Coupling}

The extended finite elements methodology is now applied to electro-mechanical coupling in one dimension. The element is divided in two sub-domains: the mechanical domain called "a" (in grey) which represents a conducting material and the electrostatic domain "b" (in white) representing a non-conducting medium. The part "b" represents, for instance, the air in which the structure moves (see Figure 3).

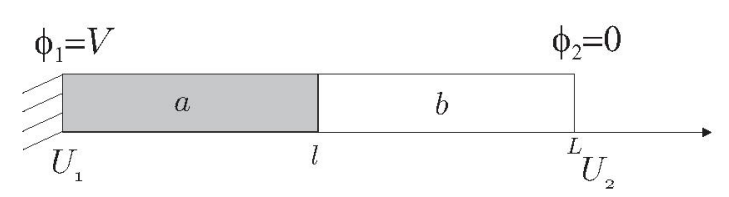

Figure 3: Extended finite element.

In electro-mechanical problems, the physical unknowns are the displacement $\boldsymbol{u}$ and the voltage $\phi$. Both fields will 
be discretised on domain $a$ and $b$. Hence we assume that there is a mechanical field and an electrostatic field in both parts and define:

$$
\left\{\begin{array}{l}
u_{a}=N_{1} U_{1}+N_{2} U_{2}+M_{1 a} A_{1}+M_{2 a} A_{2} \\
u_{b}=N_{1} U_{1}+N_{2} U_{2}+M_{1 b} A_{1}+M_{2 b} A_{2} \\
\phi_{a}=N_{1} \Phi_{1}+N_{2} \Phi_{2}+M_{1 a} B_{1}+M_{2 a} B_{2} \\
\phi_{b}=N_{1} \Phi_{1}+N_{2} \Phi_{2}+M_{1 b} B_{1}+M_{2 b} B_{2}
\end{array}\right.
$$

where $N_{i}$ are the standard shape functions, and $M_{i a}$ and $M_{i b}$ are the enriched shape functions for each domains:

$$
\left\{\begin{array} { l } 
{ N _ { 1 } = 1 - \frac { x } { L } } \\
{ N _ { 2 } = \frac { x } { L } }
\end{array} \quad \left\{\begin{array}{l}
M_{1 a}=\left(1-\frac{x}{L}\right) \frac{x}{l} \\
M_{2 a}=\frac{x}{L} \frac{x}{l} \\
M_{1 b}=\left(1-\frac{x}{L}\right) \frac{L-x}{L-l} \\
M_{2 b}=\frac{x}{L} \frac{L-x}{L-l}
\end{array}\right.\right.
$$

where $l$ is the position of the interface.

The unknowns of this problem are $U_{1}, U_{2}, \Phi_{1}$ and $\Phi_{2}$, the displacement and the electric potential at the extremities of the element. and the new unknowns $A_{1}, A_{2}, B_{1}$ and $B_{2}$ used to model the discontinuity of the mechanical field and the electric potential.

\subsubsection{Electrostatic Potential}

Along the extended element including the conductor structure and the air gap between the electrodes, the electric field is discontinuous. Indeed the voltage is contant on the conductor (mechanical part $a$ ) and decreases linearly on the electric domain $b$.

A potential difference is applied between the extremities of the element $\left(\Phi_{1}=V\right.$ and $\left.\Phi_{2}=0\right)$ as shown in Figure 3 . The stiffness matrix associated to the electrostatic field may be computed by integration over both parts of the element:

$$
\delta \boldsymbol{\Phi}^{T} \boldsymbol{K}_{\phi \phi} \delta \boldsymbol{\Phi}=\frac{1}{2} \int_{0}^{l} \frac{\partial \delta \phi}{\partial x} \varepsilon_{a} \frac{\partial \delta \phi}{\partial x} d x+\frac{1}{2} \int_{l}^{L} \frac{\partial \delta \phi}{\partial x} \varepsilon_{b} \frac{\partial \delta \phi}{\partial x} d x
$$

where $\varepsilon_{a}$ and $\varepsilon_{b}$ are the permittivity of domain $a$ and $b$, respectively. Considering the structure as a perfect conductor, the voltage on this part has to be constant. To keep the voltage constant on the mechanical part, the permittivity of this domain is imposed to be a very large number compared to the void permittivity. In this case we will take $\varepsilon_{a}=1$ and $\varepsilon_{b}=\varepsilon_{0}$.

Solving the pure electrostatic problem, the obtained potential along the element is constant on the mechanical part and decreases linearly between the electrodes as plotted in Figure 4.

\subsubsection{Electrostatic Forces at the Interface}

To compute the electrostatic forces applied at the interface between the mechanical structure and the electrostatic domain, an energetic approach has been chosen as proposed by the author in paper [4]. This method consists in determining the electrostatic forces at the nodes of a finite element by an integration on its volume. The finite element formulation is:

$$
\boldsymbol{f}_{\text {elec }}^{T} \delta \boldsymbol{u}=\frac{1}{2} \int_{\Omega} \boldsymbol{D}^{T} \boldsymbol{F}(\operatorname{grad} \delta \boldsymbol{u}) d \Omega
$$

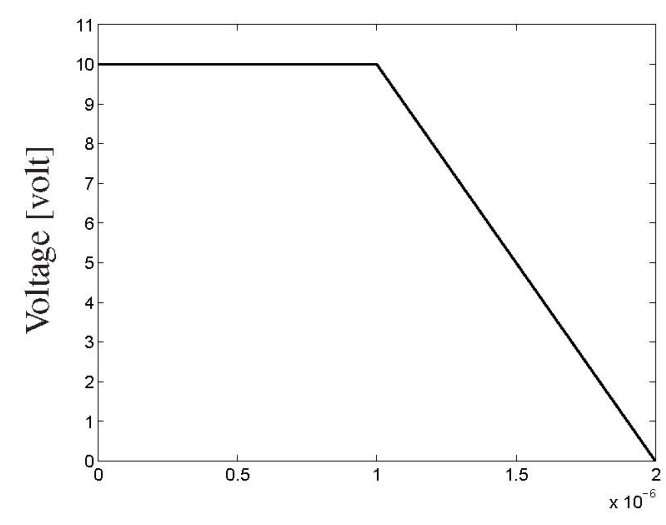

Element Length [m]

Figure 4: Electrostatic potential in one extended finite element.

with

$$
\boldsymbol{F}=\left(\begin{array}{cccc}
\frac{\partial \phi}{\partial \xi} & 0 & 2 \frac{\partial \phi}{\partial \eta} & -\frac{\partial \phi}{\partial \xi} \\
-\frac{\partial \phi}{\partial \eta} & 2 \frac{\partial \phi}{\partial \xi} & 0 & \frac{\partial \phi}{\partial \eta}
\end{array}\right)
$$

where $D$ is the electrostatic displacement. In one dimension this expression is reduced to:

$$
f_{\text {elec }}^{T} \delta u=-\frac{1}{2} \int_{0}^{L}\left(\frac{\partial \phi}{\partial x}\right)^{2} \varepsilon \frac{\partial \delta u}{\partial x} d x
$$

The same method is now applied to the extended finite element. The electrostatic forces is computed on each subdomain of the element by:

$f_{\text {elec }}^{T} \delta u=-\frac{1}{2} \int_{0}^{l}\left(\frac{\partial \phi_{a}}{\partial x}\right)^{2} \varepsilon_{a} \frac{\partial \delta u_{a}}{\partial x} d x-\frac{1}{2} \int_{l}^{L}\left(\frac{\partial \phi_{b}}{\partial x}\right)^{2} \varepsilon_{b} \frac{\partial \delta u_{b}}{\partial x} d x$

The electrostatic potential being constant on the conductor structure, the first term disappears and the electrostatic forces are computed only by the integration on domain $b$.

\subsubsection{Electro-mechanical Coupling}

Now the complete electro-mechanical problem will be considered. The mechanical stiffness of an extended onedimensional element is obtained by:

$$
\delta u^{T} \boldsymbol{K}_{u u} \delta u=\frac{1}{2} \int_{0}^{l} \frac{\partial \delta u}{\partial x} E_{a} \frac{\partial \delta u}{\partial x} d x+\frac{1}{2} \int_{l}^{L} \frac{\partial \delta u}{\partial x} E_{b} \frac{\partial \delta u}{\partial x} d x
$$

where $E_{a}$ and $E_{b}$ are the Young's modulus of the domain $a$ and $b$, respectively. In the present case, a mechanical behaviour exists only on the domain $a$ and $E_{b}$ will be set to zero.

The equilibrium position of the electro-mechanical problem may be obtained solving the system:

$$
\left\{\begin{aligned}
\boldsymbol{K}_{u u} \boldsymbol{u} & =\boldsymbol{f}_{\text {elec }} \\
\boldsymbol{K}_{\phi \phi} \boldsymbol{\Phi} & =\boldsymbol{q}_{\text {elec }}
\end{aligned}\right.
$$

where the array $\boldsymbol{u}$ contains the mechanical degrees of freedom $u_{i}$ and $A_{i}$ and $\boldsymbol{\Phi}$ contains $\Phi_{i}$ and $B_{i} . \quad q_{\text {elec }}$ are the 
charges on the nodes. This system of equation is non-linear since the electrostatic force $\boldsymbol{f}_{\text {elec }}$ is a non-linear function of the electric potential (see equation (14)) and because the position of the interface $l$ after deformation has to be taken into account in the computation of the electrostatic stiffness $\boldsymbol{K}_{\phi \phi}$ and force $\boldsymbol{f}_{\text {elec }}$.

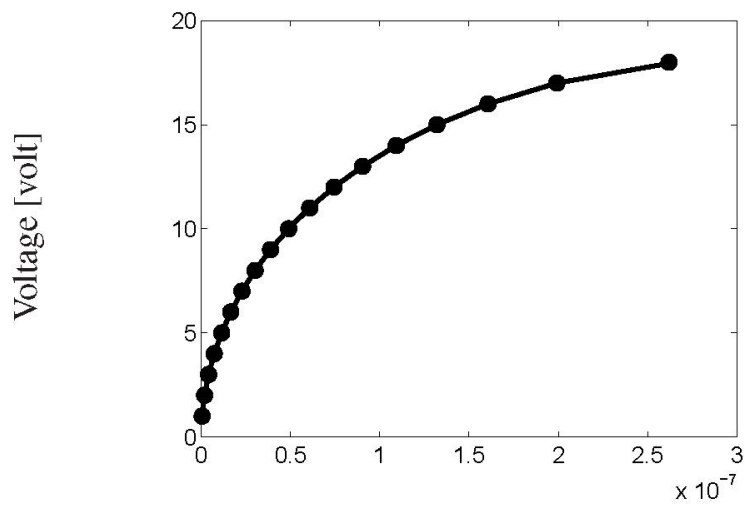

Displacement of the interface $[\mathrm{m}]$

Figure 5: Displacement of the interface (dots) and analytical solution (plain line).

In Figure 5 the displacement obtained with the extended finite element method (represented with dots) is compared to the analytical solution (plain line). These two results fit very well for the stable part of the curve.

\section{2D Electrostatic Problem}

We will now investigate possible shape functions for extending the triangular linear elements in two dimensions. Triangular elements are often used in practice in automatic meshing tools and are thus very common in practical models.

\subsection{Moes' Shape Functions}

For a triangular form Moes [2] proposes for $\theta$ the following function:

$$
\Theta=\sum_{i}\left|\psi_{i}\right| N_{i}(x, y)+\left|\sum_{i} \psi_{i} N_{i}(x, y)\right|
$$

where $\psi_{i}$ is the value of the level set of the node $i$. Let us assume that, in the reference coordinate space, the interface passes through the nodes $\left(C_{1}, C_{2}\right)$ and $\left(D_{1}, D_{2}\right)$ where $C_{1}=$ 0 and $D_{1}=1-D_{2}$ as shown in figure 6 . The level set may be chosen as:

$$
\psi(\xi, \eta)=a \xi+b \eta+c
$$

where $a=\left(D_{2}-C_{2}\right), b=-D_{1}$ and $c=C_{2} D_{1}$ so that $\psi=0$ corresponds to the equation of the interface between the two domains. Note that this choice is not unique for $a, b, c$.

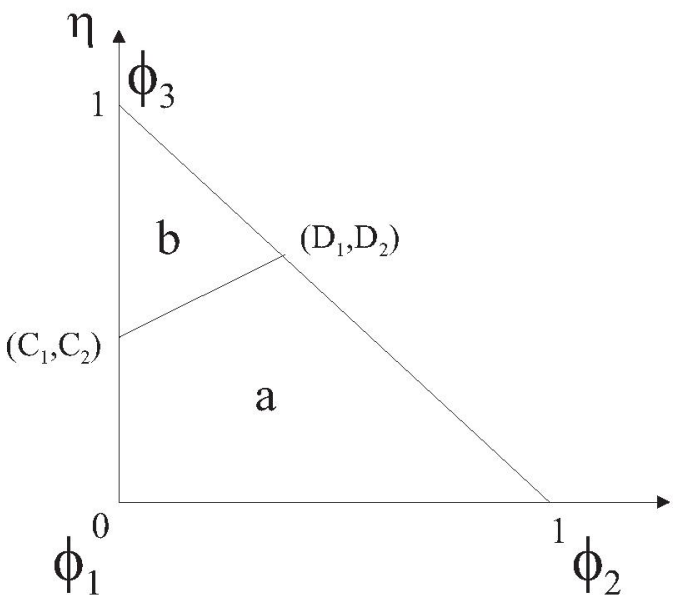

Figure 6: Extended finite element in 2D.

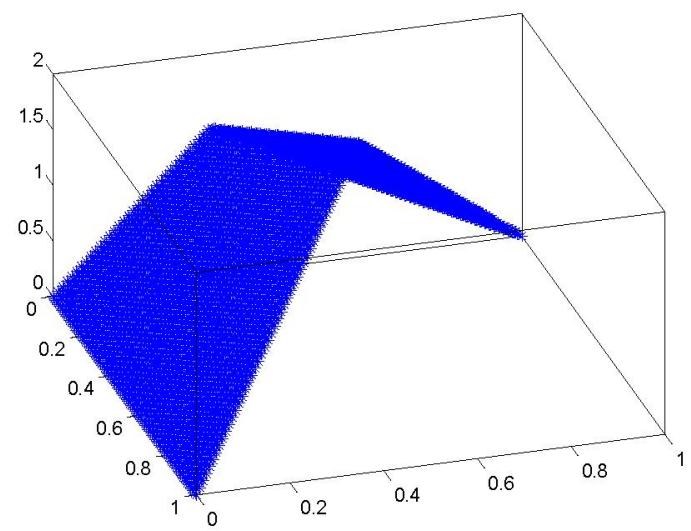

Figure 7: Enrichment hat function $\Theta$.

The enriched shape functions and the linear shape functions are:

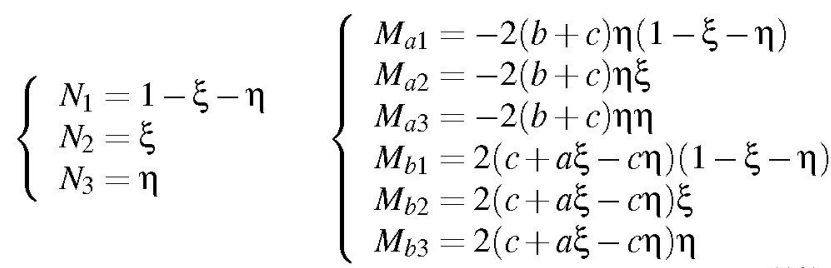

The hat function $\Theta$ modelling the discontinuity is plotted in figure 7.

$$
\Theta_{a}=\sum_{i} M_{a i} \quad \Theta_{b}=\sum_{i} M_{b i}
$$

We can observe that the line of discontinuity is not horizontal, which seems to indicate that these extended formulation might not be suitable to impose a constant potential (as needed for a conductor inside for instance). Let us check if this extended shape functions are suitable for the electrostatic problem 
So let us investigate if the extended field above can be used to model an electric potential constant on one domain and varying on the other. The potential is discretised by:

$\left\{\begin{array}{l}\phi_{a}=N_{1} \Phi_{1}+N_{2} \Phi_{2}+N_{3} \Phi_{3}+M_{1 a} B_{1}+M_{2 a} B_{2}+M_{3 a} B_{3} \\ \phi_{b}=N_{1} \Phi_{1}+N_{2} \Phi_{2}+N_{3} \Phi_{3}+M_{1 b} B_{1}+M_{2 b} B_{2}+M_{3 b} B_{3}\end{array}\right.$

Considering that the potential is constant on the quadrangular domain $a$ (see Figure 7), we impose $\frac{\partial \phi_{a}}{\partial \xi}=0$ and $\frac{\partial \phi_{a}}{\partial \eta}=0$ for all values of $\xi$ and $\eta$ on the domain $a$. After development we obtain the following constraints:

$$
\Phi_{1}=\Phi_{2} \quad \text { and } \quad B_{1}=B_{2}=B_{3}=-\frac{\left(\Phi_{1}-\Phi_{3}\right)}{2(b+c)}
$$

Only two unknowns are left, as expected, the potential $\Phi_{3}$ and $\Phi_{1}=\Phi_{2}$.

We will now consider that the potential is constant on the triangular domain $b$ so that $\frac{\partial \phi_{b}}{\partial \xi}=0$ and $\frac{\partial \phi_{b}}{\partial \eta}=0$ for all values of $\xi$ and $\eta$ on the domain $b$. The conditions to have a constant potential on this domain are

$$
B_{2}=B_{1}=B_{3} \quad \text { and } \quad \begin{aligned}
B_{1} & =\frac{\Phi_{1}-\Phi_{2}}{{ }^{2 a} \Phi_{1}} \\
B_{1} & =\frac{\Phi_{3}-\Phi_{1}}{2 c}
\end{aligned}
$$

In this case, setting the triangular domain $b$ to a constant potential $\Phi_{3}$ implies a relation between $\Phi_{1}$ and $\Phi_{2}$. This can be understood as follows.

The condition $B_{2}=B_{1}=B_{3}$ enforces the linearity of the discretisation field on domain $b$ and as a consequence, also the linearity of the field on the quadrangular domain $a$. The relation between $\Phi_{1}$ and $\Phi_{2}$ implied by the second set of constraints in (23) imposes that the values of the electric potential at the nodes "1", "2", "C" and "D" are coplanar. So the situation where $\Phi_{3}$ is set at a potential $V$ (higher electrode) and $\Phi_{1}$ and $\Phi_{2}$ are on the grounded lower electrode, is possible only if the interface is parallel to the lower electrode.

Thus it is impossible to impose a constant potential inside the triangular part of the extended element and a linear field on the quadrangular part. We must therefore conclude that the shape functions derived from the approach described in [2] not suitable for electro-mechanical modelling in the vicinity of conductors. Hence in the following section, we build a different extended field to circumvent this shortcoming.

\subsection{Quadratic Enriched Shape Functions}

The underlying idea of this new approach can be best understood by observing that if the extended element was build out of two finite elements (one for the conductor and one for the non-conducting part), it would straightforward to simulate the behaviour of electro-mechanical problem. So, we will try to use quadrangular shape functions for the trapezoidal part and triangular shape functions for the triangular part.
Therefore we will introduce two successive changes of variables corresponding to two isoparametric transformation of the physical space: a first between $(X, Y)$ and $(\xi, \eta)$, and a second between $(\xi, \eta)$ and $(s, t)$, as presented in Figure 8 . Note that the second transformation is defined separately for the triangular and the quadrangular part if the partitioned element.

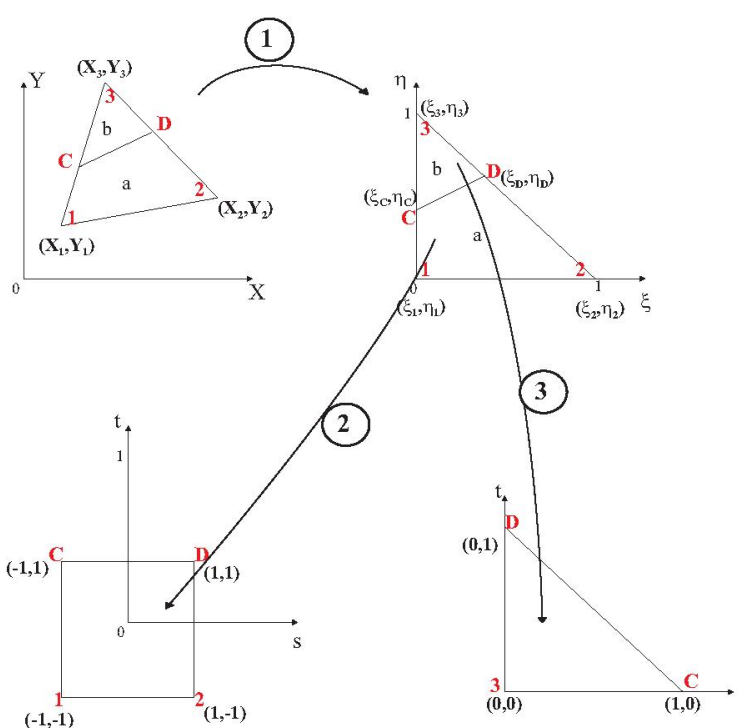

Figure 8: Successive transformations for an extended triangular element.

\subsubsection{First change of variables}

The first change of variables is identical for both domains $a$ and $b$ and may be expressed by the relation:

$$
\left\{\begin{array} { l } 
{ X = N _ { 1 } X _ { 1 } + N _ { 2 } X _ { 2 } + N _ { 3 } X _ { 3 } } \\
{ Y = N _ { 1 } Y _ { 1 } + N _ { 2 } Y _ { 2 } + N _ { 3 } Y _ { 3 } }
\end{array} \quad \left\{\begin{array}{l}
N_{1}=(1-\xi-\eta) \\
N_{2}=\xi \\
N_{3}=\eta
\end{array}\right.\right.
$$

The coordinates of the point $C$ and $D$ is obtain by computing the intersection between the level set boundary and the edge of the triangle. In the second space, the position of these points are:

$$
\left\{\begin{array} { l } 
{ \xi _ { C } = 0 } \\
{ \eta _ { C } = \frac { ( X _ { C } - X _ { 1 } ) } { ( X _ { 3 } - X _ { 1 } ) } }
\end{array} \quad \left\{\begin{array}{l}
\xi_{D}=\frac{\left(X_{3}-X_{D}\right)}{\left(X_{3}-X_{2}\right)} \\
\eta_{D}=\frac{\left(X_{D}-X_{2}\right)}{\left(X_{3}-X_{2}\right)}
\end{array}\right.\right.
$$

\subsubsection{Second Transformation - Quadrangular Part}

The usual shape functions for a quadrangle are:

$$
\left\{\begin{array}{l}
M_{1}=(1-s)(1-t) / 4 \\
M_{2}=(1+s)(1-t) / 4 \\
M_{3}=(1+s)(1+t) / 4 \\
M_{4}=(1-s)(1+t) / 4
\end{array}\right.
$$


The relation between the reference space $(s, t)$ and the intermediate space $(\xi, \eta)$ is given by the following isoparametric transformation:

$$
\left\{\begin{array}{l}
\xi(s, t)=\xi_{1} M_{1}+\xi_{2} M_{2}+\xi_{D} M_{3}+\xi_{C} M_{4} \\
\eta(s, t)=\eta_{1} M_{1}+\eta_{2} M_{2}+\eta_{D} M_{3}+\eta_{C} M_{4}
\end{array}\right.
$$

The last two shape functions will be used to enhance the solution field since the shape functions $M_{1}$ and $M_{2}$ are associated to the nodes 1 and 2 that already define the amplitude of the shape functions $N_{1}$ and $N_{2}$ in the basic element. The total discretisation of the extended field is thus:

$$
\begin{aligned}
\phi_{a}= & N_{1}(\xi(s, t), \eta(s, t)) \Phi_{1}+N_{2}(\xi(s, t), \eta(s, t)) \Phi_{2} \\
& +N_{3}(\xi(s, t), \eta(s, t)) \Phi_{3}+M_{4}(s, t) B_{C}+M_{3}(s, t) B_{D}
\end{aligned}
$$

It is easy to verify that the part of the hat function $\theta$ in domain $a$ can be found by setting $B_{C}=B_{D}$ :

$$
\Phi_{a}=M_{3}+M_{4}
$$

This is depicted in figure 9.

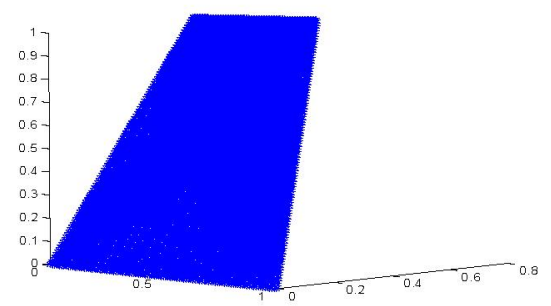

Figure 9: Enrichment of domain $a$.

Let us now compute the electric stiffness matrix. The electric stiffness matrix is computed by the relation:

$$
\boldsymbol{K}_{\mathrm{\phi} \phi_{a}}=\int_{S_{a}} \boldsymbol{B}_{a}^{T}(X, Y) \varepsilon_{a} \boldsymbol{B}_{a}(X, Y) d X d Y
$$

where $S_{a}$ is the surface of integration in the space $(X, Y)$ and where $B_{a}(X, Y)$ is the matrix of electric field shape functions obtained from the derivatives of the potential shape functions. The change of variables between the space $(X, Y)$ and the reference space $(s, t)$ may be obtained by the relation (24) and (27) which allow us to write $X(s, t), Y(s, t)$ and the Jacobian $J$ of this transformation. The electric stiffness matrix may then be computed on the reference space by:

$$
\boldsymbol{K}_{\phi, \phi_{a}}=\int_{\Omega_{a}} \boldsymbol{B}_{a}^{T}(s, t) \boldsymbol{J}^{-T} \varepsilon_{a} \boldsymbol{J}^{-1} \boldsymbol{B}_{a}(s, t) \operatorname{det}(\boldsymbol{J}) d s d t
$$

where

$$
\boldsymbol{B}_{a}(s, t)=\left(\begin{array}{lllll}
\frac{\partial N_{1}}{\partial s} & \frac{\partial N_{2}}{\partial s} & \frac{\partial N_{3}}{\partial s} & \frac{\partial M_{4}}{\partial s} & \frac{\partial M_{3}}{\partial s} \\
\frac{\partial N_{1}}{\partial t} & \frac{\partial N_{2}}{\partial t} & \frac{\partial N_{3}}{\partial t} & \frac{\partial M_{4}}{\partial t} & \frac{\partial M_{3}}{\partial t}
\end{array}\right)
$$

\subsubsection{Second Transformation - Triangular Part}

The shape functions for the triangular domain are the same as the initial change of variables:

$$
\left\{\begin{array}{l}
G_{1}=(1-s-t) \\
G_{2}=s \\
G_{3}=t
\end{array}\right.
$$

and the isoparametric transformation becomes:

$$
\left\{\begin{array}{l}
\xi(s, t)=\xi_{3} G_{1}+\xi_{C} G_{2}+\xi_{D} G_{3} \\
\eta(s, t)=\eta_{3} G_{1}+\eta_{C} G_{2}+\eta_{D} G_{3}
\end{array}\right.
$$

As for the quadrangular part only the shape function not associated to the basic node (here $G_{2}$ and $G_{3}$ ) will define the enrichment field. The total enhanced shape functions are thus, for domain $b$

$$
\begin{aligned}
\phi_{b}= & N_{1}(\xi(s, t), \eta(s, t)) \Phi_{1}+N_{2}(\xi(s, t), \eta(s, t)) \Phi_{2} \\
& +N_{3}(\xi(s, t), \eta(s, t)) \Phi_{3}+G_{2}(s, t) B_{C}+G_{3}(s, t) B_{D}
\end{aligned}
$$

Obviously since the added degrees of freedom $B_{C}$ and $B_{D}$ used here and used in the shape functions (28) in domain $a$ are identical, the continuity of the potential field is guarantied while the electric field, gradient of the potential, can be discontinuous due to the enrichment field. Again we have that the hat function $\theta$ in the extended theory is

$$
\Phi_{b}=G_{2}+G_{3}
$$

The electric stiffness matrix is computed by the relation

$$
\boldsymbol{K}_{\phi, \phi_{b}}=\int_{S_{b}} \boldsymbol{B}_{b}^{T}(X, Y) \varepsilon_{b} \boldsymbol{B}_{\boldsymbol{b}}(X, Y) d X d Y
$$

where $S_{b}$ is the surface of integration in the space $(X, Y)$. Using (24) and (34) the electric stiffness matrix may be computed on the reference space by

$$
\boldsymbol{K}_{\phi, \phi_{b}}=\int_{\Omega_{b}} \boldsymbol{B}_{b}^{T}(s, t) \boldsymbol{J}^{-T} \varepsilon_{b} \boldsymbol{J}^{-1} \boldsymbol{B}_{b}(s, t) \operatorname{det}(\boldsymbol{J}) d s d t
$$

where

$$
\boldsymbol{B}_{b}(s, t)=\left(\begin{array}{lllll}
\frac{\partial N_{1}}{\partial s} & \frac{\partial N_{2}}{\partial s} & \frac{\partial N_{3}}{\partial s} & \frac{\partial G_{2}}{\partial s} & \frac{\partial G_{3}}{\partial s} \\
\frac{\partial N_{1}}{\partial t} & \frac{\partial N_{2}}{\partial t} & \frac{\partial N_{3}}{\partial t} & \frac{\partial G_{2}}{\partial t} & \frac{\partial G_{3}}{\partial t}
\end{array}\right)
$$

\subsubsection{Simple Verification of the Element}

We will consider the very simple 2D case of a unit square domain where a voltage of $1 \mathrm{~V}$ is imposed on the top and where the lower edge is grounded. Half of the domain is conducting and the domain is modelled with 2 triangular extended elements as built in the previous section (See figure 10).

First the interface between conductor and vacuum is taken parallel to the electrodes. The computed electric potential is plotted in figure 11: it is observed that the potential is constant on the conductor and decreases linearly between the electrodes as expected. 


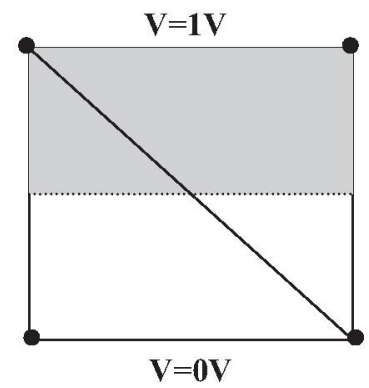

Figure 10: Simple 2D model.

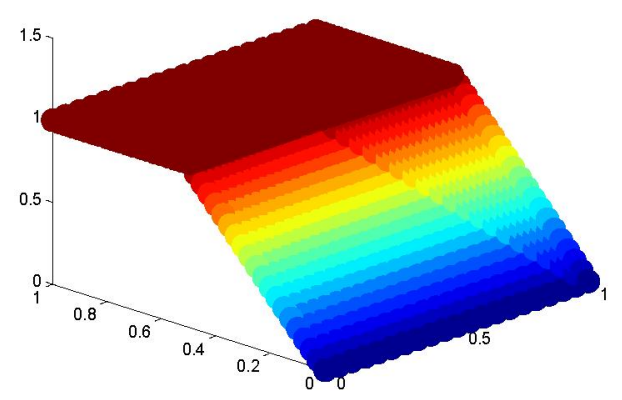

Figure 11: Electrostatic potential computed by two eXtended Elements.

Next the electric potential is also computed for the case where the interface is not parallel to the extremities as shown in figures 10 . The computed potential is shown in 13. The potential is again piecewise linear and clearly the new shape functions of the eXtended Finite Elements can properly handle the computation of the electric potential even if the interface is oblique.

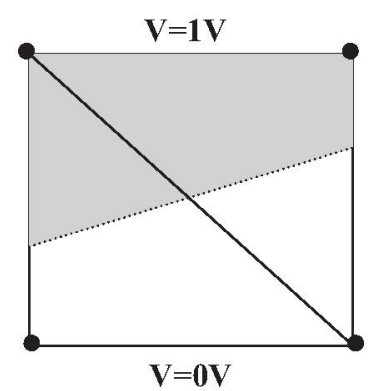

Figure 12: Simple 2D model.

\section{Conclusions}

The issue of mesh moving is a real challenge when modelling electro-mechanical devices with finite elements. This paper investigates the application of the Extended Finite Element approach to model the motion of a structure in

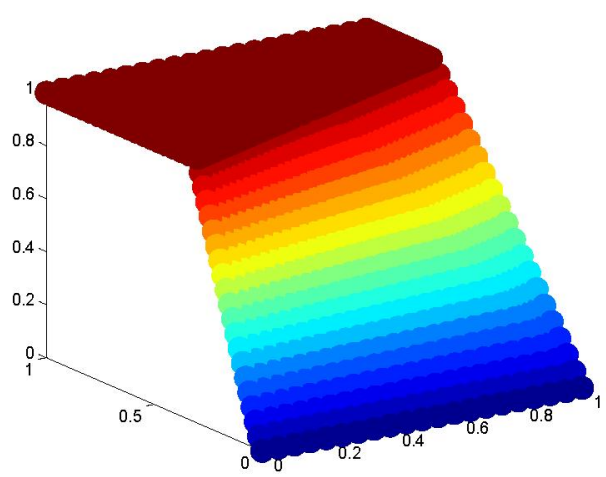

Figure 13: Electrostatic potential computed by two eXtended Elements.

an electrostatic field. The electro-mechanical forces are derived from the variational methodology proposed in paper [4].

When applied to a simple one dimensional problem, the eXtended Finite Element approach finds the exact solution for the strongly coupled electro-mechanical problem: the exact electrostatic potential along the element is retrieved and, under the action of the electrostatic forces on the interface, the correct relation between deformation and applied voltage is found.

For the two dimensional case, we have discussed why the enhancement strategy proposed in [3] is not suitable for modelling the electric field jump in practical problems. We propose in this paper different enhancement shape functions that guaranty that the potential field across a conductor/vacuum interface can be properly approximated. It is expected that the same approach can be applied in three dimensions. This will be investigate in the future together with the global efficiency of the new elements in solving the electromechanical coupling of real microsystems.

\section{Acknowledgments}

The authors want to acknowledge the support of the Koiter Institute of the Delft University of Technology and of the MicroNed program financed by the ministry of economical affairs of The Netherlands. The first author acknowledges the financial support of the Belgian National Fund for Scientific Research.

\section{References}

[1] T. Belytschko AND T. BLACK, Elastic Crack Growth in Finite Elements with Minimal Remeshing, International Journal of Numerical Methods in Engineering, Vol. 45, No. 5 (1999), pp. 601-620.

[2] N. Moës, M. Cloirec, P. Cartraud, And J. F. REMACLE, A computational approach to handle complex microstructure geometries, Computer Methods in Applied Mechanics and Engineering, Vol. 192 (2003), pp. 3163-3177. 
[3] N. Moes, J. Dolbow, and T. Belytschko, A Finite Element Method for Crack Growth without Remeshing, International Journal of Numerical Methods in Engineering, Vol. 46 (1999), pp. 131-150.

[4] V. Rochus, D. J. RiXen, AND J.-C. Golinval, Monolithical Modeling of Electro-Mechanical Coupling in Micro-Structures, International Journal for Numerical Methods in Engineering, Vol. 65, No. 4 (2006), pp. 461-493.

[5] N. Sukumar, T. Belytschko, C. PARIMI, N. MOËS, AND U. SHUJI, Modeling Holes and Inclusions by Level Sets in the Extended Finite Element Method, Computer Methods in Applied Mechanics and Engineering, Vol. 190 (2001), pp. 6183-6200. 